PRODUCTION

ENGINEERING ARCHIVES
2016, Vol. 13, No. 4, pp 44-48

ISSN 2353-5156 (print version)

ISSN 2353-7779 (online version)

Article history: $\quad$ Received: 03.12.2016 Accepted: $16.12 .2016 \quad$ Online: 30.12 .2016

\title{
Production of heterogeneous surfaces by ESD and LBM
}

\author{
Norbert Radek $^{1 *}$, Izabela Pliszka ${ }^{1}$, Bogdan Antoszewski ${ }^{1}$, Jacek Świderski $^{1}$, Jacek Pietraszek ${ }^{2}$ \\ ${ }^{1}$ University of Technology in Kielce, Al. 1000-lecia Państwa Polskiego 7, 25-314 Kielce, Poland, *norrad@tu.kielce.pl \\ ${ }^{2}$ University of Technology in Cracow, Al. Jana Pawła II, 37, 31-864 Kraków, Poland
}

Abstract. The paper is concerned with testing $\mathrm{Cu}$-Mo coatings deposited over carbon steel $\mathrm{C} 45$ which were then eroded with a laser beam. The properties were assessed by analyzing the effects of laser eroded, texturing methodology and wear tests. The tests were conducted for $\mathrm{Mo}$ and $\mathrm{Cu}$ coatings (the anode) which were electro-spark deposited over the $\mathrm{C} 45$ steel substrate (the cathode) and melted with a laser beam. The coatings were deposited by means of an ELFA-541. The laser processing was performed with an Nd:YAG laser. The coatings after laser processing are still distinguished by very good performance properties which make them suitable for use in sliding friction pairs.

Key words - heterogeneous surfaces, electro-spark deposition (ESD), laser beam machining (LBM), texturing, properties

\section{Introduction}

During tribological investigations it was found that employing heterogeneous surfaces models into boundary interaction of solid surfaces could make significant improvement (ANTOSZEWSKI B. 1999). Surfaces described as heterogeneous consist of areas which are different one from another in geometrical, physicomechanical or physicochemical properties. The heterogeneity of surfaces is frequently the result of the application of more than one technology, and can be constituted by (GYK G., ETSION I. 2006, WAN YI, XIONG DANG-SHENG 2008):

- shaped surface features such as grooves, pits or channels resulting from milling, eroding, etching, laser-beam forming, etc.,

- areas with different physicochemical and physicomechanical properties, e.g. areas with varying hardness and mechanical strength accomplished by local surfacing or selective surface hardening (e.g. electron-beam machining, laser-beam forming or thermochemical treatment),

- areas with diversified surface microgeometry, e.g. areas eroded at the points of focus (laser treatment or electro-spark deposition), or areas with formed surface microgeometry, for instance, in terms of desired microroughness directivity or load capacity (LBM and ESD technologies).

Heterogeneous surfaces can be obtained by different methods, and the laser treatment of electrospark deposited coatings is one of them. 


\section{Texture impact on contact issues}

Frictional resistance between slide rings of the endface seal depend on interrelations between elementary processes that occur in the gap (ANTOSZEWSKI B. 2010. ANTOSZEWSKI B. 2009). The processes include the following: hydrostatic and hydrodynamic action of the medium, the medium adhesion to the substrate, change in the gap geometry resulting from thermal and mechanical deformation, carrying away of heat, changes of phase and rheological properties of the liquid in the gap. Those interrelations, in turn, cannot be considered without referring to the physical properties of the medium, and of the surfaces that enclose the gap (e.g. viscosity, wettability), or to kinematic and dynamic relations between the above-mentioned factors (ARNOLD J., MUller G., SCHNEIDER H., MULlER H.K., HugEL H. 1993). It should be understood that, depending on the factors, those elementary interrelations can produce either synergistic or antagonistic effect on the minimisation of frictional resistances in the seal gap. Bearing that in mind, the role of macroand micro-geometry of the surfaces that constitute the gap needs to be taken into account. Departure from smooth and flat surface, if regular in character and properly designed, can bring about synergistic effect on the desirable properties of a sliding pair, such as bearing, durability and reliability. In view of all the arguments above, an important role for surface engineering technologies in producing sliding friction pairs should be emphasised. Those technologies, which once contributed to the manufacturing of flat and smooth surfaces, are currently employed to make heterogeneous and textured surfaces (YU X.Q., HE S. 2002, Kovalchenko A., Ajayi A., ERdemir A., Fenske G., ETISON I. 2005).

Figures 1 and 2 show examples of heterogeneous surfaces. Generally, the real contact surface is smaller than the nominal one and contact load onto real contact surfaces are higher than the corresponding nominal values. In particular, while analysing the real geometries of contact of geometrically textured surfaces, it is necessary to have data on the real contact surface (PIETRASZEK J., RADEK N., BARTKOWIAK K. 2013).

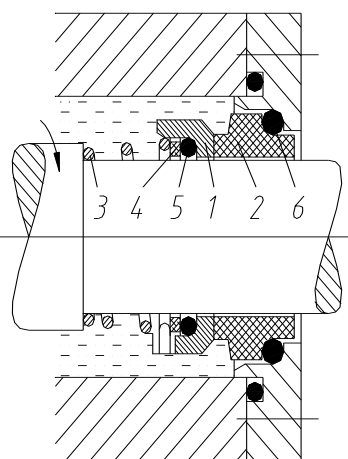

Fig. 1. Schematic diagram of the face seal: 1 -axially shifted sliding ring, 2 - anti-ring, 3 - spring, 4-clamping ring, 5, 6-secondary seals.

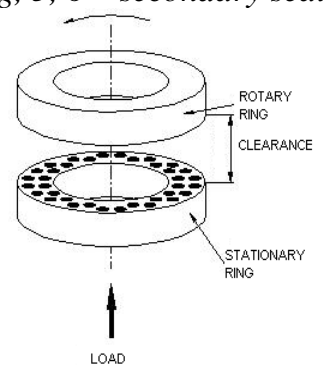

Fig. 2. Model of sliding pair with textured surface.

Depending on load, and also due to wear, the surface bearing profile undergoes changes. The analysis of changes in the bearing surface for the shape profile with spherical dimples is presented above (Fig. 3 and 4).

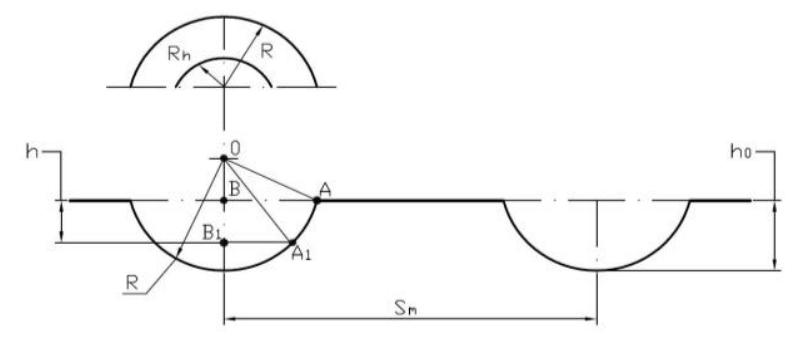

Fig. 3. Diagram of a geometric texture with spherical dimples.

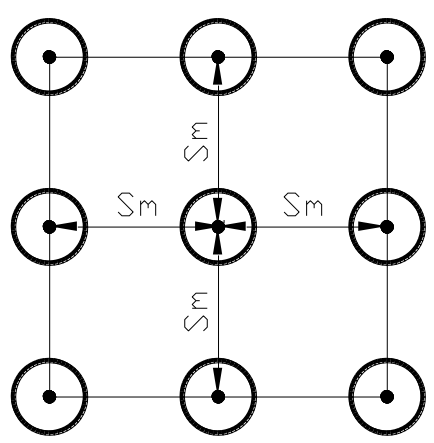

Fig. 4. Dimple spacing in the surface with uniform texture. 
As a result of deformations caused by load or wear, the depth of dimples of $h$ values is reduced in service, which also produces a reduction in the dimple radius to the value of $R_{h}$.

$$
\begin{aligned}
& \left.R_{h}=A_{1} B_{1}=\sqrt{R^{2}-\left(R-h_{0}+h\right.}\right)=\sqrt{\left(h_{0}-h\right)\left(2 R-h_{0}+h\right)} \\
& \text { For } 2 R>>h \quad R_{h}=A_{1} B_{2}=\left(R-h_{0}\right)^{\frac{1}{2}}\left(h_{0}-h\right)^{\frac{1}{2}}
\end{aligned}
$$

Because of exploitation, the bearing surface $\alpha$ (the ratio of the surface without dimples to the nominal surface) will be changed to the value of $\alpha_{h}$ :

$$
\alpha=\frac{A_{n}}{A_{0}}, \quad \alpha_{h}=\frac{A_{h}}{A_{0}}
$$

where: $A_{n}=A_{0}-k \pi R^{2}, A_{h}=A_{0}-k \pi R_{h}^{2}(k-$ number of dimples on the surface $A_{0}$ ).

If loading with $F$ force is assumed, the following dependence for the value of pressure on the surface is received:

$$
\sigma_{h}=\frac{F}{A_{h}}, \quad \sigma_{0}=\frac{F}{A_{0}}
$$

After substituting dependences (2) and (3), the following is obtained:

$$
\sigma_{h}=\left(\frac{A_{0}}{A_{0}-k \pi\left(R-h_{0}\right)\left(h_{0}-h\right)}\right) \sigma_{0}
$$

The amount of lubricant, its distribution and properties create various situations in the sliding pair. Those generate processes in the contact zone, which are different qualitatively and quantitatively. If only the amount and wetting properties of the lubricant are taken into account, four cases should be differentiated (Fig. 5).

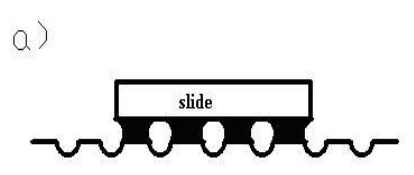

b)

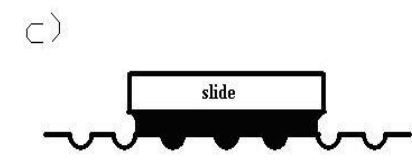

d)

Fig. 5. The interaction of the lubricant and the textured surface in the sliding pair: a) lean lubrication with a weak wetting agent, b) lean lubrication with a good wetting agent,

c) sufficient lubrication of the slide with a good wetting agent, d) abundant and continuous lubrication of the textured surface.

Texture is described by geometric relations for a single texture element, and also by relations that refer to their spacing on the friction surface. As regards a texture composed of uniformly distributed dimples having the form of spherical bowls, it is possible to state relations between a degree of blackening, diameter and depth of dimples and their volume.

\section{Texturing methodology and wear tests}

Laser surface texturing is one of the most common and promising methods of surface roughening. Categorized as a metal removal process, laser texturing is usually performed at a power density of $10^{6}-10^{9}$ $\mathrm{W} / \mathrm{cm}^{2}$. At present, it accounts for about $2 \%$ of all laser-based material processing processes used in the world.

In laser surface texturing, a pulsed laser beam is focused on a material to melt a hole. The hole depth is dependent mainly on the power density and the pulse duration. The drilling debris is removed from a hole being drilled using compressed air or another inert gas.

The tests were conducted for $\mathrm{Cu}-\mathrm{Mo}$ coatings produced by electro-spark deposition onto rings made of carbon steel C45.

The electro-spark deposition of $\mathrm{Cu}$ and Mo wires with a diameter of $1 \mathrm{~mm}$ was performed by means of an ELFA-541, a modernized device made by a Bulgarian manufacturer.

The parameters of the electro-spark deposition established during the experiment include:

- current intensity, $I=16$ A (for $\mathrm{Cu} I=8 \mathrm{~A}$ ),

- table shift rate, $v=0.5 \mathrm{~mm} / \mathrm{s}$, 
- rotational speed of the head with electrode, $n=$ $4200 \mathrm{rev} / \mathrm{min}$,

- number of coating passes, $L=2$ (for $\mathrm{Cu} L=1$ ),

- capacity of the condenser system, $C=0.47 \mu \mathrm{F}$,

- pulse duration, $t_{i}=8 \mu \mathrm{s}$,

- interpulse period, $t_{p}=32 \mu \mathrm{s}$,

- $\quad$ frequency, $f=25 \mathrm{kHz}$.

The texturing was performed using an Nd:YAG laser (impulse mode), model BLS 720, and operating in the pulse mode under the following conditions:

- laser spot diameter, $d=0.7 \mathrm{~mm}$,

- laser power, $P=20 \mathrm{~W}$,

- beam shift rate, $v=1200 \mathrm{~mm} / \mathrm{min}$,

- nozzle-sample distance, $h=1 \mathrm{~mm}$,

- pulse duration, $t_{i}=1.2 \mathrm{~ms}$,

- frequency, $f=8 \mathrm{~Hz}$.

A Joel JSM-5400 scanning electron microscope was used to study the effects of laser surface texturing. Selected SEM images are presented in Figure 6. As can be seen, the surface structure after laser surface texturing is regular. The surface is covered by bumps and dimples resulting from phase and structural modifications and the accompanying specific volume changes in the laser affected zones. Lapping and super finish are used to obtain hard flat areas transferring normal loads and areas of pores where the hydrodynamic forces are generated during fluid lubrication. Surfaces with such a texture can be applied, for instance, to sliding friction systems. The microscopic analysis showed that the removal of the drilling debris was not complete when the laser beam was focused locally. This was probably due to insufficient power density. The action of the thermocapillary forces and the convective motion resulted in the formation of rims, whose structure consisted of molten and then crystallized $\mathrm{Cu}-\mathrm{Mo}$.

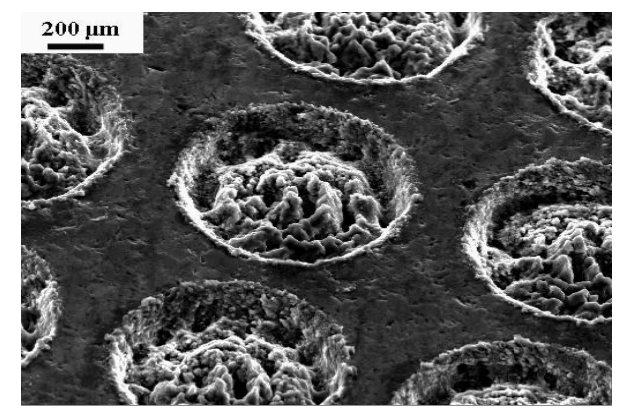

Fig. 6. A system of microcavities on the ring.
Detailed parameters of geometric texture can be identified by examining it on a profiler Talysurf FORM-120L Taylor-Hobson Limited. Figure 7 shows an example of the profile of the surface in a 3D view with the isometric map.

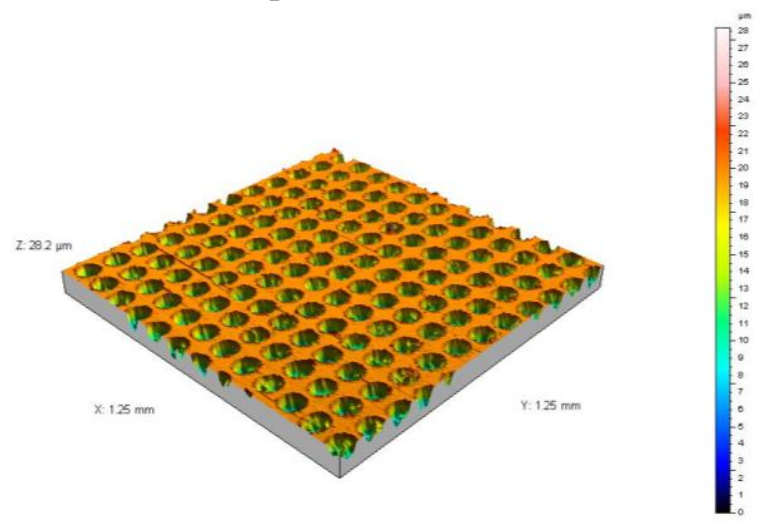

Fig. 7. Geometrical structure textured surface - 3D view.

The wear tests of the Cu-Mo electro-spark deposited coatings before and after laser surface texturing were carried out using the pin-on-disc tester T-01M.

The tester makes it possible to measure the friction force for a predetermined load. The pin $\phi 4 \times 20 \mathrm{~mm}$ was made of tool steel. The samples and anti-samples were prepared in accordance with the instruction. The tests were conducted at the following parameters of friction:

- linear velocity, $v=0.8 \mathrm{~m} / \mathrm{s}$,

- test duration, $t=3600 \mathrm{~s}$,

- sliding distance, $S=2880 \mathrm{~m}$.

- range of load changes: $5,10,15 \mathrm{~N}$.

A drop of lubricant - paraffin oil was applied on the ring raceway only once.

It was necessary to measure the time after which the value of the friction coefficient increased.

The wear test results for the electro-spark deposited $\mathrm{Cu}-\mathrm{Mo}$ coating before and after laser surface texturing are shown in Tables 1 and 2.

Table 1. Results of the wear test for the Cu-Mo coating after laser surface texturing

\begin{tabular}{|c|c|c|c|c|}
\hline \multirow{2}{*}{$\begin{array}{c}\text { Load, } \\
\mathbf{N}\end{array}$} & \multicolumn{4}{|c|}{ Mass loss, mg } \\
\cline { 2 - 5 } & \multicolumn{2}{|c|}{ not lubricated } & \multicolumn{2}{c|}{ lubricated oil } \\
\cline { 2 - 5 } & pin & disc & pin & disc \\
\hline $\mathbf{5}$ & 5.44 & 6.88 & 3.35 & 4.26 \\
\hline $\mathbf{1 0}$ & 10.24 & 12.16 & 7.11 & 8.63 \\
\hline $\mathbf{1 5}$ & 14.88 & 20.16 & 10.29 & 15.72 \\
\hline
\end{tabular}


Table 2. Results of the wear test for the Cu-Mo coating before laser surface texturing

\begin{tabular}{|c|c|c|c|c|}
\hline \multirow{2}{*}{$\begin{array}{c}\text { Load, } \\
\mathbf{N}\end{array}$} & \multicolumn{4}{|c|}{ Mass loss, mg } \\
\cline { 2 - 5 } & \multicolumn{2}{|c|}{ not lubricated } & \multicolumn{2}{c|}{ lubricated oil } \\
\cline { 2 - 5 } & pin & disc & pin & disc \\
\hline $\mathbf{5}$ & 6.16 & 9.64 & 4.89 & 6.33 \\
\hline $\mathbf{1 0}$ & 13.05 & 16.84 & 9.06 & 11.54 \\
\hline $\mathbf{1 5}$ & 19.41 & 23.28 & 13.14 & 18.67 \\
\hline
\end{tabular}

Table 3 shows the values of the friction coefficient for the $\mathrm{Cu}$-Mo coating before and after laser surface texturing.

Table 3. Results of the friction coefficient for the $\mathrm{Cu}$-Mo coating before and after laser surface texturing

\begin{tabular}{|c|c|c|c|c|}
\hline \multirow{2}{*}{$\begin{array}{c}\text { Load, } \\
\mathbf{N}\end{array}$} & \multicolumn{4}{|c|}{ Friction coefficient } \\
\cline { 2 - 5 } & \multicolumn{2}{|c|}{ not lubricated } & \multicolumn{2}{c|}{ lubricated oil } \\
\cline { 2 - 5 } & Cu-Mo & CuMo+laser & Cu-Mo & Cu-Mo+laser \\
\hline $\mathbf{5}$ & 0.39 & 0.40 & 0.21 & 0.14 \\
\hline $\mathbf{1 0}$ & 0.54 & 0.46 & 0.35 & 0.23 \\
\hline $\mathbf{1 5}$ & 0.67 & 0.48 & 0.43 & 0.32 \\
\hline
\end{tabular}

\section{Summary}

1. It is possible to diversify the surface of electrospark deposited coatings, i.e. to obtain heterogeneous surfaces. The laser-affected areas are characterized by the occurrence of regular cavities, hardened areas and varied roughness.

2. The surface heterogeneity (i.e. the cavities) is desirable in sliding friction pairs. They may be used as reservoirs of lubricants as well as sources of hydrodynamic forces increasing the capacity of a sliding pair.

3. Laser texturing of $\mathrm{Cu}-\mathrm{Mo}$ coatings provides increased resistance to wear and reducing of friction coefficient.

4. A concentrated laser beam can effectively modify the state of the surface layer, i.e. the functional properties of electro-spark coatings can be achieved.

5. In the next phase of the research it is essential to determine the phase composition and porosity of the coatings before and after laser treatment.

\section{Literature}

1. AntoszeWSKI B. 1999. Influence of laser and plasma modification on surface properties of sliding and rubbing components. Wydawnictwo Politechniki Świętokrzyskiej, Kielce.

2. GYK G., ETSION I. 2006. Testing piston rings with partial laser surface texturing for friction reduction. Vol. 216 Wear.

3. WAN YI, XIONG DANG-SHENG 2008. The effect of laser surface texturing on frictional performance of face seal. Vol. 197 Journal of Materials Processing Technology.

4. AntoszeWsKi B. 2010. The surface layers of texture the formation of selected technologies bundled and tribological properties. Wydawnictwo Politechniki Świętokrzyskiej, Kielce.

5. AntoszewsKi B. 2010. Problems of maintenance of sustainable technological systems. [In:] Improvement of the conditions of lubrication friction ties by laser textured lubrication, Wydawnictwo PNTTE 2010, Tom 1.

6. ANTOSZEWSKI B. 2009. The formation of antiwear surface layers on elements of machine parts. Vol. 44 No. 2, Zagadnienia Eksploatacji Maszyn.

7. Arnold J., Muller G., Schneider H., Muller H. K., Hugel H. 1993. Production of micro structures in SiC slide rings with an excimer laser. Vol. 25 Laser und Optoelektronik.

8. ETSION I. 2003. A laser surface textured hydrostatic mechanical seal sealing technology Vol. 3 Sealing Technology.

9. YU X. Q., HE S. 2002. Frictional characteristics of mechanical seals with a laser textured seal face. Vol. 129 Journal of Materials Processing Technology.

10. Kovalchenko A., Ajayi A., Erdemir A., Fenske G., ETISON I. 2005. The effect of laser surface texturing on transitions in lubrication regimes during unidirectional sliding contact. Vol. 38 Tribology International.

11. PietraszeK J., RADEK N., BARTKOWIAK K. 2013. Advanced statistical refinement of surface layer's discretization in the case of electro-spark deposited carbide-ceramic coatings modified by a laser beam. Vol. 197 Solid State Phenomena. 